\title{
Direct test of time-reversal symmetry in the entangled neutral kaon system at a $\phi$-factory
}

\section{A. Di Domenico*}

Department of Physics, Sapienza University of Rome, and INFN Sezione di Roma, P.le A. Moro, 2, I-00185 Rome, Italy

E-mail: antonio.didomenico@romal.infn.it

\begin{abstract}
A novel method to perform a direct $\mathrm{T}$ (time reversal) symmetry test in the neutral kaon system is presented, independent of any CP and/or CPT symmetry tests. This is based on the comparison of suitable transition probabilities, where the required interchange of in $\leftrightarrow$ out states for a given process is obtained exploiting the Einstein-Podolsky-Rosen correlations of neutral kaon pairs produced at a $\phi$-factory.

The statistical significance of the test achievable with the KLOE-2 experiment at DAФNE, the Frascati $\phi$-factory, is also briefly discussed, together with the possibility to perform a novel CPT test, similarly to the proposed $\mathrm{T}$ test.
\end{abstract}

2013 Kaon Physics International Conference,

29 April-1 May 2013

University of Michigan, Ann Arbor, Michigan - USA

${ }^{*}$ Speaker. 


\section{Introduction}

In the context of a local quantum field theory with Lorentz invariance and Hermiticity, as in the Standard Model, the validity of the CPT theorem ensures an automatic theoretical connection between any source of $\mathrm{CP}$ violation and a corresponding $\mathrm{T}$ (time reversal) violation. Even though CPT invariance has been confirmed by all present experimental tests, particularly in the neutral kaon system with stringent limits to possible CPT violation effects $[1,3,4,5,6]$, the theoretical connection between $\mathrm{CP}$ and $\mathrm{T}$ symmetries does not imply an experimental identity between them. In fact a direct evidence of $\mathrm{T}$ violation should result from an experiment clearly showing the violation, independently from the results of $\mathrm{CP}$ violation and without any connection with them.

In case of a transition process, due to the antiunitarity of the operator implementing the symmetry transformation, $\mathrm{T}$ invariance requires that the rate for the reaction $i \rightarrow f$ equals that of the reaction $f_{T} \rightarrow i_{T}$, with in and out states exchanged and $\mathrm{T}$ inverted (for spinless particles this corresponds to the reaction $f \rightarrow i$ ).

In the past the measurement of a non-zero value of the Kabir asymmetry [7], comparing the rates of the process $\mathrm{K}^{0} \rightarrow \overline{\mathrm{K}}^{0}$ and its T conjugated one $\overline{\mathrm{K}}^{0} \rightarrow \mathrm{K}^{0}$, has been presented as a proof for $\mathrm{T}$ violation $[8,9]$. However, this process has the feature that $\mathrm{K}^{0} \rightarrow \overline{\mathrm{K}}^{0}$ is a CPT even transition, so that $\mathrm{T}$ and $\mathrm{CP}$ transformations are identical in this case, and the corresponding observables are not independent. Therefore it is impossible to separate $\mathrm{T}$ violation from $\mathrm{CP}$ violation in the Kabir asymmetry.

Another subtle drawback is the one discussed in Ref. [10], where it is pointed out that the Tviolation contribution in the Kabir asymmetry is constant in time and directly proportional to $\Delta \Gamma$, the width difference of the $\mathrm{K}_{\mathrm{S}, \mathrm{L}}$ mass eigenstates. Therefore $\mathrm{T}$ non-invariance would not be present in the limit $\Delta \Gamma \rightarrow 0$, as one might like in a direct genuine test. In the $\mathrm{B}^{0}-\overline{\mathrm{B}}^{0}$ system no asymmetry is expected to be found, since in this case $\Delta \Gamma$ almost vanishes (within the $\mathrm{SM}$ ).

In order to overcome these difficulties it has been suggested to exploit the Einstein-PodolskyRosen (EPR) [11] entanglement of neutral mesons produced at a $\phi$-factory (or B-factory) [12, 13, 14]. In fact in this case other transitions than $\mathrm{K}^{0} \rightarrow \overline{\mathrm{K}}^{0}$ or $\overline{\mathrm{K}}^{0} \rightarrow \mathrm{K}^{0}$ can be observed, independently from $\mathrm{CP}$ or $\mathrm{CPT}$ test results, thus implementing a genuine test of the T symmetry.

Recently, the first direct observation of $\mathrm{T}$ violation, in this sense, has been accomplished in the neutral B meson system [15]. In the present paper the methodology to perform a direct test of $\mathrm{T}$ symmetry in the neutral kaon system at a $\phi$-factory is described ${ }^{1}$, which presents some notable differences with respect to the B meson case [16]. The statistical significance of the test achievable with the KLOE-2 experiment at DAФNE, the Frascati $\phi$-factory [17], is also briefly discussed, together with the possibility to perform a novel CPT test, similarly to the proposed T test (see detailed discussion in Appendix A of Ref. [16], and also Ref.[18]).

\section{Observables at a $\phi$-factory}

The initial state of the neutral kaon pair produced in $\phi \rightarrow \mathrm{K}^{0} \overline{\mathrm{K}}^{0}$ decay can be rewritten in terms

\footnotetext{
${ }^{1}$ It is based on a work in collaboration with J. Bernabeu and P. Villanueva [16], developing an original idea of J. Bernabeu.
} 
of any pair of orthogonal states $\left|\mathrm{K}_{+}\right\rangle$and $\left|\mathrm{K}_{-}\right\rangle$:

$$
|i\rangle=\frac{1}{\sqrt{2}}\left\{\left|\mathrm{~K}^{0}\right\rangle\left|\overline{\mathrm{K}}^{0}\right\rangle-\left|\overline{\mathrm{K}}^{0}\right\rangle\left|\mathrm{K}^{0}\right\rangle\right\}=\frac{1}{\sqrt{2}}\left\{\left|\mathrm{~K}_{+}\right\rangle\left|\mathrm{K}_{-}\right\rangle-\left|\mathrm{K}_{-}\right\rangle\left|\mathrm{K}_{+}\right\rangle\right\}
$$

Here one can consider the states $\left|\mathrm{K}_{+}\right\rangle,\left|\mathrm{K}_{-}\right\rangle$defined as follows: $\left|\mathrm{K}_{+}\right\rangle$is the state filtered by the decay into $\pi \pi\left(\pi^{+} \pi^{+}\right.$or $\left.\pi^{0} \pi^{0}\right)$, a pure $\mathrm{CP}=+1$ state; analogously $\left|\mathrm{K}_{-}\right\rangle$is the state filtered by the decay into $3 \pi^{0}$, a pure $\mathrm{CP}=-1$ state. Their orthogonal states correspond to the states which cannot decay into $\pi \pi$ or $3 \pi^{0}$, defined, respectively, as

$$
\begin{aligned}
\left|\widetilde{\mathrm{K}}_{-}\right\rangle & \propto\left[\left|\mathrm{K}_{\mathrm{L}}\right\rangle-\eta_{\pi \pi}\left|\mathrm{K}_{\mathrm{S}}\right\rangle\right] \\
\left|\widetilde{\mathrm{K}}_{+}\right\rangle & \propto\left[\left|\mathrm{K}_{\mathrm{S}}\right\rangle-\left(\eta_{3 \pi^{0}}^{-1}\right)\left|\mathrm{K}_{\mathrm{L}}\right\rangle\right],
\end{aligned}
$$

with $\eta_{\pi \pi}=\left\langle\pi \pi|T| \mathrm{K}_{\mathrm{L}}\right\rangle /\left\langle\pi \pi|T| \mathrm{K}_{\mathrm{S}}\right\rangle$ and $\left(\eta_{3 \pi^{0}}^{-1}\right)=\left\langle 3 \pi^{0}|T| \mathrm{K}_{\mathrm{S}}\right\rangle /\left\langle 3 \pi^{0}|T| \mathrm{K}_{\mathrm{L}}\right\rangle$. With these definitions of states, it can be shown that the condition of orthogonality $\left\langle\mathrm{K}_{-} \mid \mathrm{K}_{+}\right\rangle=0$, (i.e. $\left|\mathrm{K}_{+}\right\rangle \equiv\left|\widetilde{\mathrm{K}}_{+}\right\rangle$and $\left|\mathrm{K}_{-}\right\rangle \equiv\left|\widetilde{\mathrm{K}}_{-}\right\rangle$) corresponds to assume negligible direct CP violation (or CPT violation in decay) contributions (i.e. $\varepsilon^{\prime}, \varepsilon_{000}^{\prime} \ll \varepsilon$ ), assumption quite well satisfied for neutral kaons (see detailed discussion in Appendix A of Ref. [16]). The validity of the $\Delta S=\Delta Q$ rule is also assumed, so that the two flavor orthogonal eigenstates $\left|\mathrm{K}^{0}\right\rangle$ and $\left|\overline{\mathrm{K}}^{0}\right\rangle$ are identified by the charge of the lepton in semileptonic decays, i.e. a $\left|\mathrm{K}^{0}\right\rangle$ can decay into $\pi^{-} \ell^{+} v$ and not into $\pi^{+} \ell^{-} \bar{v}$, and vice-versa for a $\left|\overline{\mathrm{K}}^{0}\right\rangle$.

Thus, exploiting the perfect anticorrelation of the states implied by Eq. (2.1), it is possible to have a "flavor-tag" or a "CP-tag", i.e. to infer the flavor $\left(\mathrm{K}^{0}\right.$ or $\left.\overline{\mathrm{K}}^{0}\right)$ or the $\mathrm{CP}\left(\mathrm{K}_{+}\right.$or $\left.\mathrm{K}_{-}\right)$state of the still alive kaon by observing a specific flavor decay $\left(\pi^{+} \ell^{-} \nu\right.$ or $\left.\pi^{-} \ell^{+} \bar{v}\right)$ or CP decay $(\pi \pi$ or $3 \pi^{0}$ ) of the other (and first decaying) kaon in the pair.

In this way one can experimentally access other transitions than $\mathrm{K}^{0} \rightarrow \overline{\mathrm{K}}^{0}$ or $\overline{\mathrm{K}}^{0} \rightarrow \mathrm{K}^{0}$. These new accessible processes can be divided into four categories of events, as summarized in Table 1.

\begin{tabular}{c|c|c|c}
\hline Reference & T-conjug. & CP-conjug. & CPT-conjug. \\
\hline $\mathrm{K}^{0} \rightarrow \mathrm{K}_{+}$ & $\mathrm{K}_{+} \rightarrow \mathrm{K}^{0}$ & $\overline{\mathrm{K}}^{0} \rightarrow \mathrm{K}_{+}$ & $\mathrm{K}_{+} \rightarrow \overline{\mathrm{K}}^{0}$ \\
$\mathrm{~K}^{0} \rightarrow \mathrm{K}_{-}$ & $\mathrm{K}_{-} \rightarrow \mathrm{K}^{0}$ & $\overline{\mathrm{K}}^{0} \rightarrow \mathrm{K}_{-}$ & $\mathrm{K}_{-} \rightarrow \overline{\mathrm{K}}^{0}$ \\
$\overline{\mathrm{K}}^{0} \rightarrow \mathrm{K}_{+}$ & $\mathrm{K}_{+} \rightarrow \overline{\mathrm{K}}^{0}$ & $\mathrm{~K}^{0} \rightarrow \mathrm{K}_{+}$ & $\mathrm{K}_{+} \rightarrow \mathrm{K}^{0}$ \\
$\overline{\mathrm{K}}^{0} \rightarrow \mathrm{K}_{-}$ & $\mathrm{K}_{-} \rightarrow \overline{\mathrm{K}}^{0}$ & $\mathrm{~K}^{0} \rightarrow \mathrm{K}_{-}$ & $\mathrm{K}_{-} \rightarrow \mathrm{K}^{0}$ \\
\hline
\end{tabular}

Table 1: Scheme of possible reference transitions and their associated T, CP or CPT conjugated processes accessible at a $\phi$-factory.

For instance, one can consider $\mathrm{K}^{0} \rightarrow \mathrm{K}_{+}$as the reference process, where the initial state $\mathrm{K}^{0}$ is identified at time $t_{1}$ with the flavor tag, and the final state $\mathrm{K}_{+}$is identified at a subsequent time $t_{2} \geq t_{1}$ with a CP decay.

I) The $\mathrm{T}$ transformed process is $\mathrm{K}_{+} \rightarrow \mathrm{K}^{0}$; any asymmetry in the rate between $\mathrm{K}^{0} \rightarrow \mathrm{K}_{+}$and $\mathrm{K}_{+} \rightarrow \mathrm{K}^{0}$ would be a genuine $\mathrm{T}$ violating effect.

II) The $\mathrm{CP}$ transformed process is $\overline{\mathrm{K}}^{0} \rightarrow \mathrm{K}_{+}$; any asymmetry in the rate between $\mathrm{K}^{0} \rightarrow \mathrm{K}_{+}$and $\overline{\mathrm{K}}^{0} \rightarrow \mathrm{K}_{+}$would be a genuine CP violating effect. 
III) The CPT transformed process is $\mathrm{K}_{+} \rightarrow \overline{\mathrm{K}}^{0}$; any asymmetry in the rate between $\mathrm{K}^{0} \rightarrow \mathrm{K}_{+}$and $\mathrm{K}_{+} \rightarrow \overline{\mathrm{K}}^{0}$ would be a genuine CPT violating effect.

One may check that the events used for the asymmetries in I), II), and III) are completely independent from each other. Moreover, $\mathrm{T}$ violating effects would depend on $\Delta t=t_{2}-t_{1}$ and would be present even in the limit $\Delta \Gamma \rightarrow 0$.

\section{T symmetry test}

For the direct $\mathrm{T}$ symmetry test one can define the following ratios of probabilities:

$$
\begin{aligned}
& R_{1}(\Delta t)=P\left[\mathrm{~K}^{0}(0) \rightarrow \mathrm{K}_{+}(\Delta t)\right] / P\left[\mathrm{~K}_{+}(0) \rightarrow \mathrm{K}^{0}(\Delta t)\right] \\
& R_{2}(\Delta t)=P\left[\mathrm{~K}^{0}(0) \rightarrow \mathrm{K}_{-}(\Delta t)\right] / P\left[\mathrm{~K}_{-}(0) \rightarrow \mathrm{K}^{0}(\Delta t)\right] \\
& R_{3}(\Delta t)=P\left[\overline{\mathrm{K}}^{0}(0) \rightarrow \mathrm{K}_{+}(\Delta t)\right] / P\left[\mathrm{~K}_{+}(0) \rightarrow \overline{\mathrm{K}}^{0}(\Delta t)\right] \\
& R_{4}(\Delta t)=P\left[\overline{\mathrm{K}}^{0}(0) \rightarrow \mathrm{K}_{-}(\Delta t)\right] / P\left[\mathrm{~K}_{-}(0) \rightarrow \overline{\mathrm{K}}^{0}(\Delta t)\right] .
\end{aligned}
$$

The measurement of any deviation from the prediction $R_{i}(\Delta t)=1$ imposed by $\mathrm{T}$ invariance is a signal of $\mathrm{T}$ violation.

At a $\phi$-factory the observable quantity is the double differential decay rate of the state $|i\rangle$ into decay products $f_{1}$ and $f_{2}$ at proper times $t_{1}$ and $t_{2}$, respectively [19]:

$$
\begin{array}{r}
I\left(f_{1}, t_{1} ; f_{2}, t_{2}\right)=C_{12}\left\{\left|\eta_{1}\right|^{2} e^{-\Gamma_{L} t_{1}-\Gamma_{S} t_{2}}+\left|\eta_{2}\right|^{2} e^{-\Gamma_{S} t_{1}-\Gamma_{L} t_{2}}\right. \\
\left.-2\left|\eta_{1}\right|\left|\eta_{2}\right| e^{-\frac{\left(\Gamma_{S}+\Gamma_{L}\right)}{2}\left(t_{1}+t_{2}\right)} \cos \left[\Delta m\left(t_{1}-t_{2}\right)+\phi_{2}-\phi_{1}\right]\right\}
\end{array}
$$

with

and

$$
\begin{gathered}
\eta_{i} \equiv\left|\eta_{i}\right| e^{i \phi_{i}}=\frac{\left\langle f_{i}|T| \mathrm{K}_{\mathrm{L}}\right\rangle}{\left\langle f_{i}|T| \mathrm{K}_{\mathrm{S}}\right\rangle}, \\
C_{12}=\frac{|\mathscr{N}|^{2}}{2}\left|\left\langle f_{1}|T| \mathrm{K}_{\mathrm{S}}\right\rangle\left\langle f_{2}|T| \mathrm{K}_{\mathrm{S}}\right\rangle\right|^{2},
\end{gathered}
$$

a normalization factor.

Let us consider two generic orthogonal basis, $\left\{\mathrm{K}_{\mathbf{X}}, \overline{\mathrm{K}}_{\mathrm{X}}\right\}$ and $\left\{\mathrm{K}_{\mathrm{Y}}, \overline{\mathrm{K}}_{\mathrm{Y}}\right\}$ (in our case $\left\{\mathrm{K}^{0}, \overline{\mathrm{K}}^{0}\right\}$ or $\left\{\mathrm{K}_{+}, \mathrm{K}_{-}\right\}$), for which the decays $\mathrm{K}_{\mathrm{X}} \rightarrow f_{\bar{X}}$ and $\overline{\mathrm{K}}_{\mathrm{Y}} \rightarrow f_{Y}$ are forbidden while the decays $\overline{\mathrm{K}}_{\mathrm{X}} \rightarrow f_{\bar{X}}$ and $\mathrm{K}_{\mathrm{Y}} \rightarrow f_{Y}$ are allowed.

After integration on $t_{1}$ at fixed time difference $\Delta t=t_{2}-t_{1}>0$, the decay intensity (3.2) can be rewritten in a more suitable form, putting in evidence the probabilities we are aiming for. In particular it will be a function of the first decay product $f_{1}=f_{\bar{X}}$ (which takes place at time $t_{1}$, identifies a $\overline{\mathrm{K}}_{\mathrm{X}}$ state, and tags a $\mathrm{K}_{\mathrm{X}}$ state on the opposite side), and the second decay product $f_{2}=f_{Y}$ (which takes place at time $t_{2}$ and identifies a $\mathrm{K}_{\mathrm{Y}}$ state):

$$
\begin{aligned}
I\left(f_{\bar{X}}, f_{Y} ; \Delta t\right) & =\int_{0}^{\infty} I\left(f_{\bar{X}}, t_{1} ; f_{Y} ; t_{2}\right) d t_{1} \\
& =\frac{1}{\Gamma_{S}+\Gamma_{L}}\left|\left\langle\mathrm{~K}_{X} \overline{\mathrm{K}}_{\mathrm{X}} \mid i\right\rangle\left\langle f_{\bar{X}}|T| \overline{\mathrm{K}}_{\mathrm{X}}\right\rangle\left\langle\mathrm{K}_{\mathrm{Y}} \mid \mathrm{K}_{X}(\Delta t)\right\rangle\left\langle f_{Y}|T| \mathrm{K}_{\mathrm{Y}}\right\rangle\right|^{2} \\
& =C\left(f_{\bar{X}}, f_{Y}\right) \times P\left[\mathrm{~K}_{\mathrm{X}}(0) \rightarrow \mathrm{K}_{\mathrm{Y}}(\Delta t)\right],
\end{aligned}
$$


where the coefficient $C\left(f_{\bar{X}}, f_{Y}\right)$, depending only on the final states $f_{\bar{X}}$ and $f_{Y}$, is given by:

$$
C\left(f_{\bar{X}}, f_{Y}\right)=\frac{1}{2\left(\Gamma_{S}+\Gamma_{L}\right)}\left|\left\langle f_{\bar{X}}|T| \overline{\mathrm{K}}_{\mathrm{X}}\right\rangle\left\langle f_{Y}|T| \mathrm{K}_{\mathrm{Y}}\right\rangle\right|^{2} .
$$

As a consequence at a $\phi$-factory the experimentally observable quantities corresponding to the four ratios $R_{i}(\Delta t)$ are two ratios of decay intensities (3.2): $R_{2}^{\exp }(\Delta t)$ and $R_{4}^{\exp }(\Delta t)$ (see Fig.1). They are are defined and connected to the ratios $R_{i}(\Delta t)$ as follows. For $\Delta t>0$ one has:

$$
R_{2}^{\exp }(\Delta t) \equiv \frac{I\left(\ell^{-}, 3 \pi^{0} ; \Delta t\right)}{I\left(\pi \pi, \ell^{+} ; \Delta t\right)}=R_{2}(\Delta t) \times D, R_{4}^{\exp }(\Delta t) \equiv \frac{I\left(\ell^{+}, 3 \pi^{0} ; \Delta t\right)}{I\left(\pi \pi, \ell^{-} ; \Delta t\right)}=R_{4}(\Delta t) \times D
$$

while for $\Delta t<0$ :

$$
R_{2}^{\exp }(\Delta t)=\frac{D}{R_{3}(|\Delta t|)}, R_{4}^{\exp }(\Delta t)=\frac{D}{R_{1}(|\Delta t|)} .
$$

Here the normalization constant $D$, assuming no CPT violation in semileptonic decays, is $D=$ $\left\{\mathrm{BR}\left(\mathrm{K}_{\mathrm{L}} \rightarrow 3 \pi^{0}\right) \cdot \Gamma_{L}\right\} /\left\{\mathrm{BR}\left(\mathrm{K}_{\mathrm{S}} \rightarrow \pi \pi\right) \cdot \Gamma_{S}\right\}$.

The KLOE-2 experiment at DA $\Phi$ NE with an integrated luminosity of $\mathscr{O}\left(10 \mathrm{fb}^{-1}\right)$ [17] could make a statistically significant T test, measuring the ratios $R_{2}^{\exp }(\Delta t)$ and $R_{4}^{\exp }(\Delta t)$ integrated in the statistically most populated $\Delta t$ region, $0 \leq \Delta t \leq 300 \tau_{S}$ [16]. In this region the uncertainty on the knowledge of the $\left(\eta_{3 \pi^{0}}^{-1}\right)$ parameter, which corresponds to the main uncertainty on the validity of the orthogonality condition $\left\langle\mathrm{K}_{-} \mid \mathrm{K}_{+}\right\rangle=0$, is negligible, as shown in Figs.1,2 (see detailed discussion in Ref.[16]). Moreover in this region $R_{2}^{\exp }(\Delta t)$ and $R_{4}^{\exp }(\Delta t)$ are expected to be constant (see Fig. 2), and a precise knowledge of the normalization $D$ is needed in order to detect $\mathrm{T}$ violation. This also implies that $\mathrm{T}$ violation in this $\Delta t$ region is constant, proportional to $\Re \varepsilon$, i.e. directly proportional to $\Delta \Gamma$. Therefore, in this $\Delta t$ region, $\mathrm{T}$ violation would not be present in the limit $\Delta \Gamma \rightarrow 0$.

\section{CPT symmetry test}

Similarly to the proposed $\mathrm{T}$ test, one can define the following ratios of probabilities for a CPT test:

$$
\begin{aligned}
& R_{1, \mathrm{CPT}}(\Delta t)=P\left[\mathrm{~K}^{0}(0) \rightarrow \mathrm{K}_{+}(\Delta t)\right] / P\left[\mathrm{~K}_{+}(0) \rightarrow \overline{\mathrm{K}}^{0}(\Delta t)\right] \\
& R_{2, \mathrm{CPT}}(\Delta t)=P\left[\mathrm{~K}^{0}(0) \rightarrow \mathrm{K}_{-}(\Delta t)\right] / P\left[\mathrm{~K}_{-}(0) \rightarrow \overline{\mathrm{K}}^{0}(\Delta t)\right] \\
& R_{3, \mathrm{CPT}}(\Delta t)=P\left[\overline{\mathrm{K}}^{0}(0) \rightarrow \mathrm{K}_{+}(\Delta t)\right] / P\left[\mathrm{~K}_{+}(0) \rightarrow \mathrm{K}^{0}(\Delta t)\right] \\
& R_{4, \mathrm{CPT}}(\Delta t)=P\left[\overline{\mathrm{K}}^{0}(0) \rightarrow \mathrm{K}_{-}(\Delta t)\right] / P\left[\mathrm{~K}_{-}(0) \rightarrow \mathrm{K}^{0}(\Delta t)\right]
\end{aligned}
$$

The measurement of any deviation from the prediction $R_{i, \mathrm{CPT}}(\Delta t)=1$ imposed by CPT invariance is a signal of CPT violation.

At a $\phi$-factory the corresponding observable quantities are, for $\Delta t>0$ :

$$
\begin{aligned}
& R_{2, \mathrm{CPT}}^{\exp }(\Delta t) \equiv \frac{I\left(\ell^{-}, 3 \pi^{0} ; \Delta t\right)}{I\left(\pi \pi, \ell^{-} ; \Delta t\right)}=R_{2, \mathrm{CPT}}(\Delta t) \times D_{\mathrm{CPT}} \\
& R_{4, \mathrm{CPT}}^{\exp }(\Delta t) \equiv \frac{I\left(\ell^{+}, 3 \pi^{0} ; \Delta t\right)}{I\left(\pi \pi, \ell^{+} ; \Delta t\right)}=R_{4, \mathrm{CPT}}(\Delta t) \times D_{\mathrm{CPT}}
\end{aligned}
$$



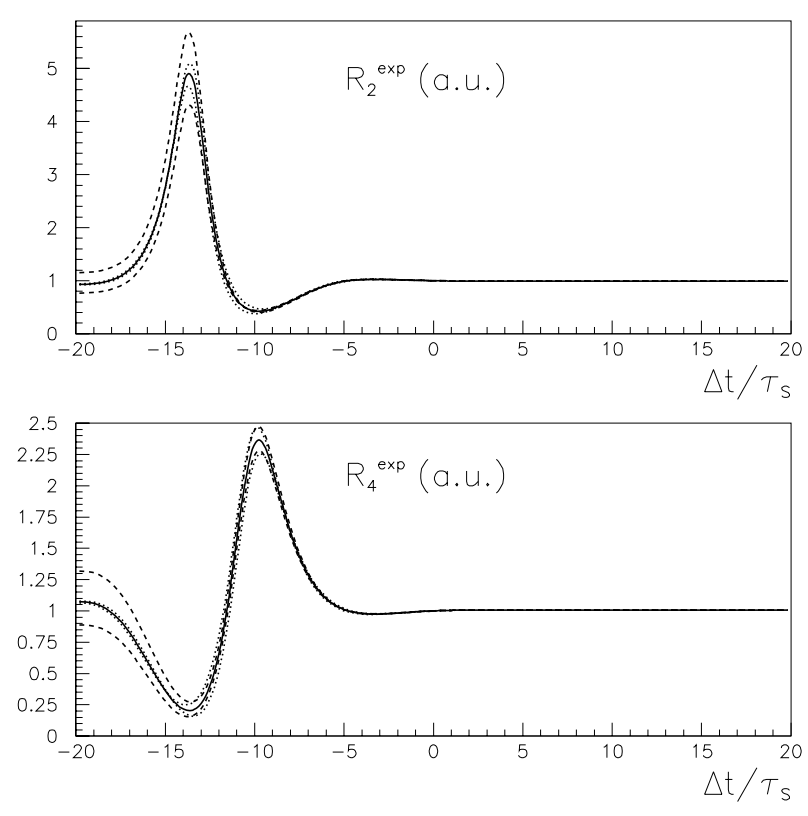

Figure 1: The expected ratios $R_{2}^{\exp }(\Delta t)$ (top) and $R_{4}^{\exp }(\Delta t)$ (bottom) as a function of $\Delta t$ (solid line); dashed lines correspond to $\pm 10 \%$ uncertainty on modulus and $\pm 10^{\circ}$ on phase of $\left(\eta_{3 \pi^{0}}^{-1}\right)$; the constant $D$ has been fixed to one for simplicity.

while for $\Delta t<0$ :

$$
R_{2, \mathrm{CPT}}^{\exp }(\Delta t)=\frac{D_{\mathrm{CPT}}}{R_{1, \mathrm{CPT}}(|\Delta t|)}, R_{4, \mathrm{CPT}}^{\exp }(\Delta t)=\frac{D_{\mathrm{CPT}}}{R_{3, \mathrm{CPT}}(|\Delta t|)} .
$$

Here the normalization constant $D_{\mathrm{CPT}}$ is $D_{\mathrm{CPT}}=\left\{\mathrm{BR}\left(\mathrm{K}_{\mathrm{L}} \rightarrow 3 \pi^{0}\right) \cdot \Gamma_{L}\right\} /\left\{\mathrm{BR}\left(\mathrm{K}_{\mathrm{S}} \rightarrow \pi \pi\right) \cdot \Gamma_{S}\right\}$ without any assumption on CPT violation in semileptonic decays.

The KLOE-2 experiment could make a statistically significant CPT test, measuring the ratios $R_{2, \mathrm{CPT}}^{\exp }(\Delta t)$ and $R_{4, \mathrm{CPT}}^{\exp }(\Delta t)$ integrated in the statistically most populated $\Delta t$ region, $0 \leq \Delta t \leq 300 \tau_{S}$ $[16,18]$. In this region $R_{2, \mathrm{CPT}}^{\exp }(\Delta t)$ and $R_{4, \mathrm{CPT}}^{\exp }(\Delta t)$ are expected to be constant, and in particular one has:

$$
\begin{aligned}
& R_{2, \mathrm{CPT}}^{\exp }\left(\Delta t \gg \tau_{S}\right)=(1-4 \mathfrak{R} \delta) \times D_{\mathrm{CPT}} \\
& R_{4, \mathrm{CPT}}^{\exp }\left(\Delta t \gg \tau_{S}\right)=(1+4 \mathfrak{R} \delta) \times D_{\mathrm{CPT}}
\end{aligned}
$$




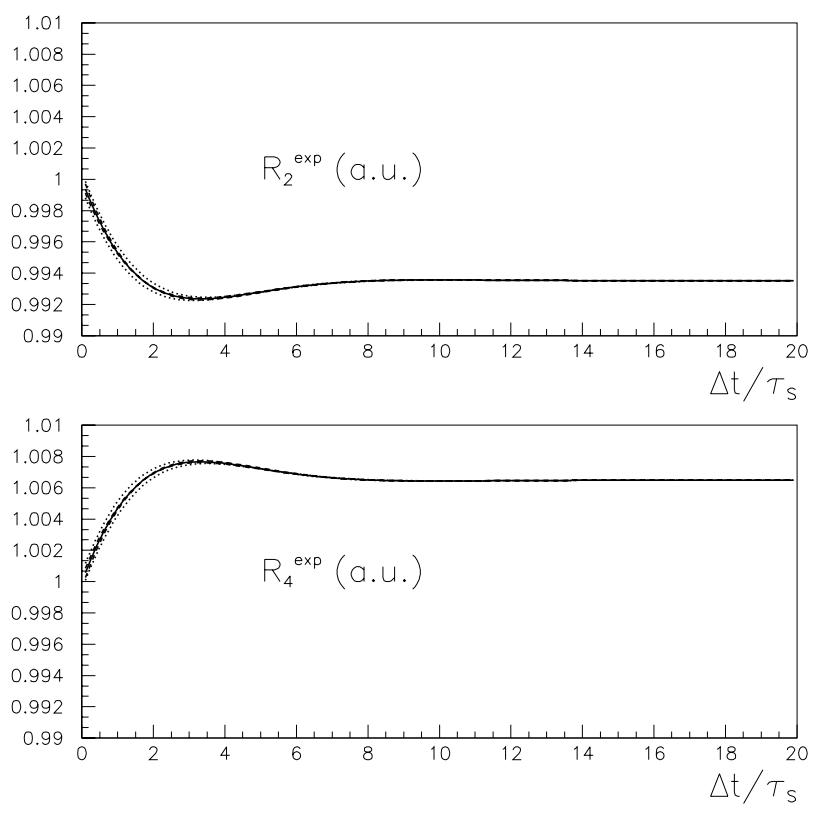

Figure 2: Same as in Fig. 1 but with zoomed plots in the region $\Delta t>0$.

with $\delta$ the usual CPT violation parameter in the effective Hamiltonian of the neutral kaon system. In this case CPT violation is proportional to $\Re \delta$, which do not vanish in the limit $\Delta \Gamma \rightarrow 0$, escaping the previous controversy for the $\mathrm{T}$ test. Moreover the impact of the uncertainty on the knowledge of $\left(\eta_{3 \pi^{0}}^{-1}\right)$ is negligible in this $\Delta t$ region (see Figs.3, 4), and would not appreciably affect the significance of the CPT test.

\section{Acknowledgements}

I am indebted to J. Bernabeu for several illuminating discussions on the subject. I would like to thank Monica Tecchio, Yau Wah, and all the organizing committee for the invitation and the pleasant stay in Ann Arbor.

\section{References}

[1] See "CPT invariance tests in neutral kaon decay" and "Tests of conservations laws" reviews in [2]. 

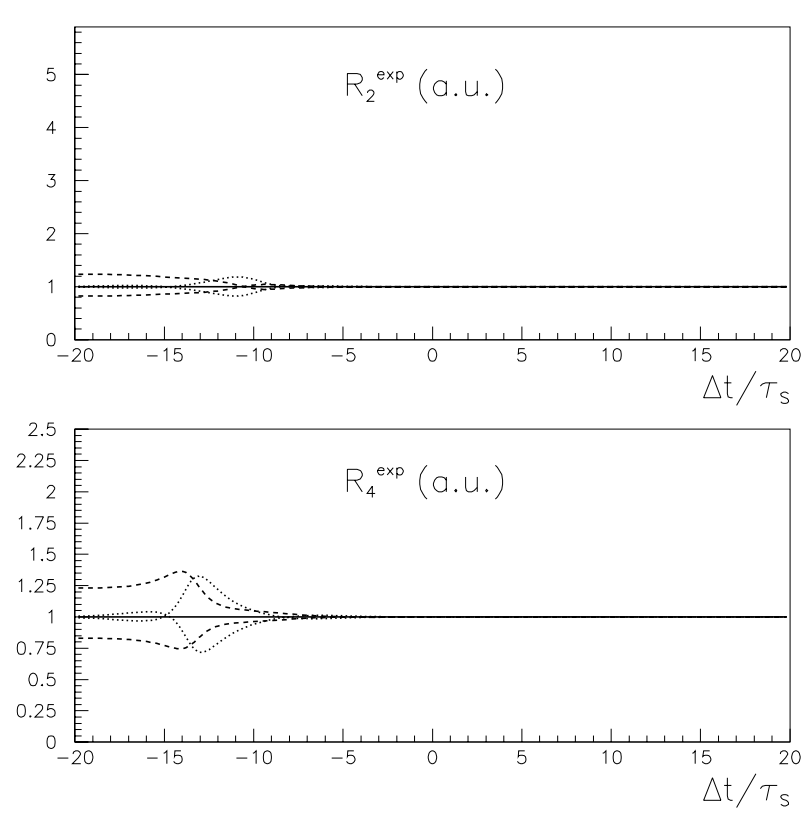

Figure 3: The expected ratios $R_{2, \mathrm{CPT}}^{\exp }(\Delta t)$ (top) and $R_{4, \mathrm{CPT}}^{\exp }(\Delta t)$ (bottom) (with the CPT violation parameter $\delta$ fixed to zero) as a function of $\Delta t$ (solid line); dashed lines correspond to $\pm 10 \%$ uncertainty on modulus and $\pm 10^{\circ}$ on phase of $\left(\eta_{3 \pi^{0}}^{-1}\right)$; the constant $D_{\mathrm{CPT}}$ has been fixed to one for simplicity.

[2] J. Beringer et al., Particle Data Group, Phys. Rev. D 86, 010001 (2012).

[3] F. Ambrosino et al., KLOE collaboration, JHEP 12011 (2006).

[4] F. Ambrosino et al., KLOE collaboration, Phys. Lett. B 642, 315 (2006).

[5] A. Di Domenico and the KLOE collaboration, J. Phys. Conf. Ser. 171, 012008 (2009).

[6] A. Di Domenico and the KLOE collaboration, Found. Phys. 40 , 852 (2010).

[7] P. K. Kabir, Phys. Rev. D 2, 540 (1970).

[8] A. Angelopoulos et al., CPLEAR coll., Phys. Lett. B 444, 43 (1999).

[9] A. Angelopoulos et al., CPLEAR coll., Eur. Phys. C 22, 55 (2001).

[10] L. Wolfenstein, Int. J. Mod. Phys. E 8, 501 (1999).

[11] A. Einstein, B. Podolski and N. Rosen, Phys. Rev. 47, 777 (1935). 


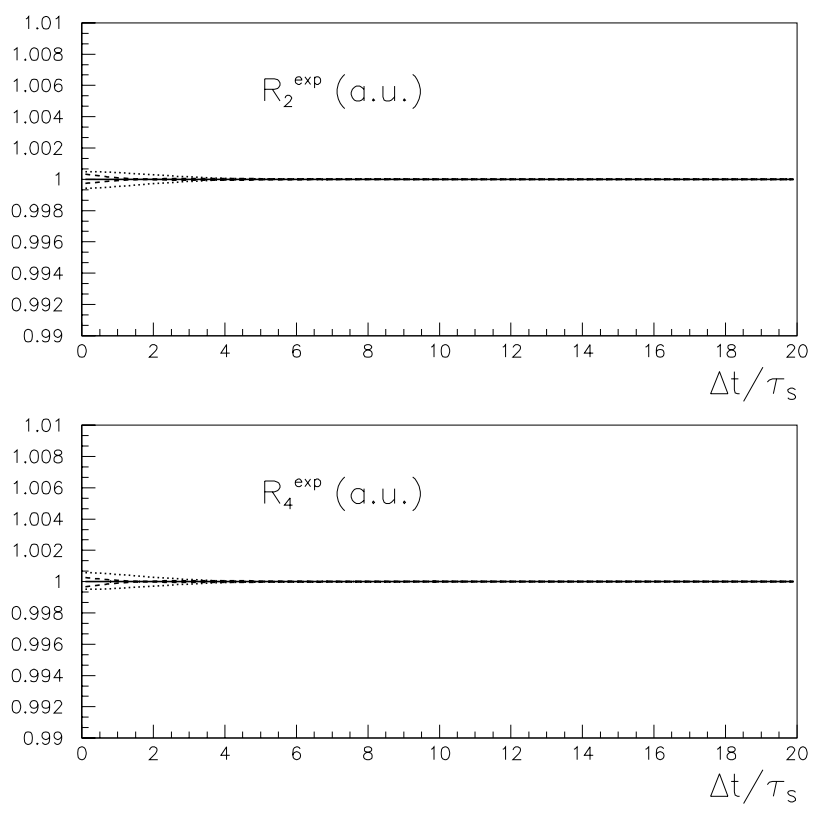

Figure 4: Same as in Fig. 3 but with zoomed plots in the region $\Delta t>0$.

[12] M. C. Banuls and J. Bernabeu, Phys. Lett. B 464, 117 (1999); Nucl. Phys. B 590, 19 (2000).

[13] J. Bernabeu, J. Phys. Conf. Ser. 335012011 (2011).

[14] J. Bernabeu, F. Martínez-Vidal, P. Villanueva-Pérez, JHEP 1208064 (2012).

[15] J. P. Lees et al., BABAR Collaboration, Phys. Rev. Lett. 109211801 (2012).

[16] J. Bernabeu, A. Di Domenico, P. Villanueva-Perez, Nucl. Phys. B 868102 (2013).

[17] G. Amelino-Camelia et al., Eur. Phys. J. C 68, 619 (2010).

[18] A. Di Domenico, to appear in proceedings of Workshop on T violation and CPT tests in neutral-meson systems, Mainz April 15-16, 2013.

[19] A. Domenico et al. , Handbook on Neutral Kaon Interferometry at a $\phi$-factory, Frascati Phys. Ser. 43 (2007). 